decreased in analogous rate in all groups. 2 patients in LAM+ADV and ETV groups developed VB. No viral mutations associated with drug resistance were detected in the LAM + ADV and TDFgroup, including those with VB, NR or SR; in contrast 1 ETV patient with $\mathrm{SR}$ at m12 (genotype C) developed mutation rtM204I. HBeAg seroconversion was more frequent in LAM + ADV cohort vs ETV and TDF (21\% vs $8 \%$ and $7 \%, \mathrm{p}=0.06)$ and $\mathrm{HBsAg}$ seroconversion occurred only in LAM + ADV and ETV patients ( $\%$ and $1 \%)$.

Conclusion De-novo antiviral therapy with different therapeutic approaches of nucleos(t)ide analogues LAM+ADV, ETV and TDF achieves similar efficacy within 12 months of treatment in real-life patient cohorts with $\mathrm{CH}-\mathrm{B}$

\section{P48 TELAPREVIR SUBSTANTIALLY IMPROVED SVR RATES ACROSS ALL IL28B GENOTYPES IN THE ADVANCE TRIAL}

doi:10.1136/gutjnl-2011-300857a.48

${ }^{1} \mathrm{G} M$ Dusheiko, ${ }^{2} \mathrm{M}$ Jacobson, ${ }^{3} \mid$ Catlett, ${ }^{3} \mathrm{~S}$ George, ${ }^{3} \mathrm{~S}$ Seepersaud, ${ }^{3} \mathrm{R}$ Ramachandran, ${ }^{3} \mathrm{~K}$ Sussky, ${ }^{3} \mathrm{R}$ S Kauffman, ${ }^{3} \mathrm{M}$ Botfield. ${ }^{1}$ Royal Free and University College, London, UK; ${ }^{2}$ Weill Cornell Medical College, New York, New York, USA; ${ }^{3}$ Vertex Pharmaceuticals Incorporated, Cambridge, Massachusetts, USA

Aim Single nucleotide polymorphisms (SNPs) near the IL28B gene region have been strongly associated with the likelihood of SVR in genotype $1 \mathrm{HCV}$ patients treated with peginterferon/ribavirin (PR). During the evaluation of an exploratory diagnostic test that characterises genetic polymorphisms near the IL28B gene, the impact of rs1297860 on SVR in telaprevir (T)-based regimens in the ADVANCE trial was evaluated.

Method IL28B genotype testing was performed according to a US FDA guidance governing use of de-identified leftover samples for in vitro diagnostic testing. The guidance requires a strict de-identification procedure that was carried out by an independent third party. Only specimens from the USA were used; and as nonCaucasian patients could not be de-identified in sufficient numbers, they were excluded from the study.

Results The diagnostic assay developed provided consistent, unambiguous genotype calls and was considered suitable for research. 454/1088 (42\%) patients had IL28B test results available. 150/454 (33\%) were CC, 224/454 (49\%) CT, and 80/454 (18\%) TT. SVR rates for each subgroup by arm are shown in the Abstract P48 table $1.72 \%, 54 \%$ and $48 \%$ of CC, CT and TT telaprevir patients, respectively had undetectable HCV RNA at weeks 4 and 12 (eRVR) compared with $16 \%, 2 \%$ and $0 \%$ of PR patients. Among eRVR telaprevir patients, $91 \%$ achieved SVR (97\% of CC, $88 \%$ of CT, $85 \%$ of TT) with 24 weeks of therapy whereas $43 \%$ of non-eRVR telaprevir patients had SVR ( $63 \%$ of CC, $33 \%$ of CT, $46 \%$ of TT) with 48 weeks of therapy.

\section{Abstract P48 Table 1 SVR rates}

\begin{tabular}{|c|c|c|c|c|c|}
\hline \multirow[b]{2}{*}{$\%(n / N)$} & \multicolumn{4}{|c|}{ In patients tested for IL28B allele } & \multirow{2}{*}{$\begin{array}{l}\text { In all ADVANCE } \\
\text { patients } \\
(\mathbf{N}=1088)\end{array}$} \\
\hline & $C C(N=150)$ & CT $(N=224)$ & $\mathrm{TT}(\mathrm{N}=\mathbf{8 0})$ & Total $(\mathrm{N}=454)$ & \\
\hline T12PR* & $90(45 / 50)$ & $71(48 / 68)$ & $73(16 / 22)$ & $78(109 / 140)$ & $75(271 / 363)$ \\
\hline T8PR** & $84(38 / 45)$ & $57(43 / 76)$ & $59(19 / 32)$ & $65(100 / 153)$ & $69(250 / 364)$ \\
\hline PR & $64(35 / 55)$ & $25(20 / 80)$ & $23(6 / 26)$ & $38(61 / 161)$ & $44(158 / 361)$ \\
\hline
\end{tabular}

${ }^{*} \mathrm{~T} 12 \mathrm{PR}=\mathrm{T}+\mathrm{PR} 12$ weeks, then PR 12 or 36 weeks depending on eRVR status.

${ }^{* *} \mathrm{~T} 8 \mathrm{PR}=\mathrm{T}+\mathrm{PR} 8$ weeks, then PR 16 or 40 weeks depending on eRVR status.
Conclusion Telaprevir-based therapy improved eRVR and SVR rates across all IL28B genotypes. Specifically, telaprevir-based therapy more than doubled the rates of SVR in CT/TT patients, and substantially increased SVR rates in those with CC genotype, as compared with PR therapy alone. Non-attainment of eRVR was associated with lower SVR rates across all IL28B genotypes, with the largest decrement in CT/TT patients.

\section{P49 TREATING HEPATITIS C IN THE PATIENT'S HOME: A HOSPITAL AND HOMECARE PARTNERSHIP}

doi:10.1136/gutjnl-2011-300857a.49

${ }^{1} \mathrm{~K}$ Jack, ${ }^{2} \mathrm{~J}$ Barnett, ${ }^{1} \mathrm{~B}$ Thomson. ${ }^{1}$ Nottingham University Hospitals NHS Trust ${ }^{2}$ Healthcare At Home

Introduction It is long established that the UK has poorer outcomes regarding numbers of patients treated with antivirals for chronic hepatitis C (HCV) than it's European counterparts. Exploring alternative models of care that will facilitate the engagement of those whom regular hospital attendance would be a barrier to treatment is important if one is to reduce the incidence of end stage liver disease among this group of patients. Against this backdrop we initiated a project in Nottingham to deliver care in tandem with a community nursing service.

Aim To treat HCV infected patients with pegylated interferon and ribavirin in the patient's home via a partnership between secondary care and an established homecare company.

Method Patients with stable HCV infection and no evidence of decompensated liver disease are offered this model of care in the hepatitis clinic and referred to the homecare company by the Consultant or Specialist Nurse. The antiviral drugs are delivered directly to the patient's home, and a skilled homecare nurse trained in the management of HCV visits the patient to undertake: teaching how to self inject pegylated interferon and take ribavirin correctly; draw blood samples for monitoring treatment progress and safety, to assess side effects and provide nursing care in managing these; and regularly report back to the referring clinician. Nursing support is available to patients $24 \mathrm{~h}$ a day. Once treatment is complete the patient returns to the hepatitis clinic to be reviewed.

Results Since this model's inception in February 2004, approximately 110 patients were offered the option of homecare. 87 patients elected to be treated at home and were referred by the secondary care HCV clinic using an agreed proforma. Investigations during treatment were conducted using the same schedule as the specialist clinic and hospital staff reviewed the results. The specialist team took all decisions on changes to drug treatment. Treatment outcomes and drop out rates are comparable to hospital-managed clinics, but the non-attendance rates are exceptionally low; only two home visits have been missed by patients. No adverse events as a result of receiving treatment and monitoring at home have occurred. Furthermore this model of care is cost effective; drugs are supplied VAT-free by not being routed into the hospital pharmacy, and this offsets the home nursing cost.

Conclusion Our results demonstrate that homecare treatment for HCV infection is feasible, safe and the preferred option of most patients. It is well tolerated by patients with very high compliance rates which we anticipate will lead to improvements in treatment outcomes. We suggest that this innovative homecare model can be an important facet of hospital HCV services, and thus be a major means of facilitating the engagement of more patients into therapy without an additional burden of nursing staff costs. 